\title{
Role of NKG2D in cytokine-induced killer cells against lung cancer
}

\author{
XIAOWEI YIN ${ }^{1}$, XUZHANG LU ${ }^{2}$, ZHANG XIUWEN ${ }^{2}$, ZHOU MIN $^{3}$, \\ RONG XIAO $^{2}$, ZHENGDAO MAO $^{1}$ and QIAN ZHANG ${ }^{1}$ \\ Departments of ${ }^{1}$ Respiratory Medicine and ${ }^{2}$ Hematology, \\ Changzhou No. 2 People's Hospital Affiliated to Nanjing Medical University; \\ ${ }^{3}$ Department of Hematology, Changzhou No. 3 People's Hospital, Changzhou, Jiangsu 213000, P.R. China
}

Received August 18, 2015; Accepted January 19, 2017

DOI: $10.3892 / \mathrm{ol} .2017 .5800$

\begin{abstract}
It has been previously demonstrated that cytokine-induced killer (CIK) cells possess potent cytotoxicity against various cancer cells, including lung cancer cells. However, the mechanism by which CIK cells recognize lung cancer cells has not been understood. The interaction between killer cell lectin like receptor $\mathrm{K} 1$ (NKG2D) receptor and NKG2D ligands was demonstrated to serve an important role in target cell killing by natural killer cells. The present study investigated whether NKG2D receptor and NKG2D ligand interactions are involved in the CIK-directed killing of lung cancer cells. The expression of MICA and ULBP2 was detected in tumor and healthy tissue samples. The expression of MICA and ULBP2 in tumor tissue samples was higher compared with that in the healthy control tissue. The expression of NKG2D ligands was analyzed in A549 and Q56 cells through reverse transcription-quantitative polymerase chain reaction and flow cytometry. The results demonstrated that the lung cancer cell lines markedly expressed the NKG2D ligands. Furthermore, NKG2D ligand-expressing lung cancer cells were targeted by CIK cells, which was partially blocked by treating CIK cells with an antibody against NKG2D. The data of the current study has demonstrated that the NKG2D-NKG2D ligand interaction serves an essential role in mediating lung cancer cell killing by CIK cells.
\end{abstract}

\section{Introduction}

Lung cancer is the most commonly diagnosed cancer and also the leading cause of cancer-associated mortality (1). Even

Correspondence to: Dr Xiaowei Yin, Department of Respiratory Medicine, Changzhou No. 2 People's Hospital Affiliated to Nanjing Medical University, 29 Xinglong Alley, Changzhou, Jiangsu 213000 , P.R. China

E-mail: yjj1401@163.com

Key words: NKG2D ligand, cytokine-induced killer cells, lung cancer, MICA/B with more advanced chemotherapeutic agents and molecularly targeted drugs, the prognosis of this disease remains poor due to limited treatment efficacy $(2,3)$. Previously, maintenance therapy has been identified to be an acceptable treatment paradigm to improve progression free survival (4). However, data from randomized clinical trials have demonstrated that the maintenance and consolidation therapy failed to improve the outcomes of patients with lung cancer, and in certain cases caused severe side effects or toxicity-associated mortality (5). Thus, given the higher recurrence and mortality rates, novel therapeutic strategies are warranted in order to improve the outcome of patients with lung cancer.

Aimed at eliminating tumor cells through stimulation or restoration of a patient's immune system, adoptive cellular immunotherapies have attracted increasing interests. Among them, considerable attention has been given to cytokine induced killer (CIK) cells derived from peripheral blood for treating various types of cancer (6). Killer cell lectin like receptor K1 (NKG2D) has been demonstrated to serve an important role in mediating the elimination of tumor cells by cytotoxic effectors cells (7). Previous studies have demonstrated that effector cell recognition and the lysis of tumor cells are primarily mediated through NKG2D activating receptor (8-10). NKG2D-mediated cytotoxicity depends on immune cell surface expression of NKG2D receptors and target cell expression of NKG2D ligands (11). Previous studies have demonstrated that increased expression of NKG2D ligands sensitizes target cells to natural killer (NK) cell-mediated lysis (12-14). There are two categories of NKG2D ligands (15), including MHC class I polypeptide-related sequence (MIC) A and B, and UL16 binding protein (ULBP) 1, 2 and 3. It was indicated that multiple malignancies, including primary leukemia, glioma, and melanoma tumors, expressed ULBP, and the expression of MICA and ULBP1-3 were identified in almost all primary glioma isolates, but little expression of MICB on primary glioma was detected (16-18). Therefore, NKG2D is important in tumor immune surveillance to prevent tumor initiation and in immunotherapy. In the present study, the expression of NKG2D ligands in samples from patients with lung cancer, and in A549 and Q56 cells was investigated. The cytotoxicity of CIK cells against A549 cell was subsequently analyzed. The current study aimed to investigate the mechanisms underlying 
the effects of CIK cells in tumor cell elimination, in order to improve the efficacy of CIK cell therapy in the clinical practice.

\section{Materials and methods}

Patients. The present study conformed to The Declaration of Helsinki and was approved by the Institute Review Committee of the No. 2 People's Hospital of Changzhou (Changzhou, China). Written informed consent was obtained from all participants. Patients with lung cancer were identified from the surgical pathology biorepository of the Department of Thoracic Surgery at the No. 2 People's Hospital of Changzhou. No other specific inclusion or exclusion criteria were applied to the present study. Healthy controls were recruited from individuals who visited the No. 2 People's Hospital of Changzhou for a routine health check-up without any history of cancer.

Cell lines. The lung cancer cell lines A549 and QG56 were purchased from The Cell Bank of Type Culture Collection of Chinese Academy of Sciences (Shanghai, China). Cells were maintained in Dulbecco's modified Eagle medium (DMEM, HyClone; GE Healthcare Life Sciences, Logan, UT, USA) supplemented with $100 \mathrm{IU} / \mathrm{ml}$ penicillin, $100 \mu \mathrm{g} / \mathrm{ml}$ streptomycin and $10 \%$ fetal bovine serum (FBS; HyClone; GE Healthcare Life Sciences). The cells were incubated at $37^{\circ} \mathrm{C}$ with $5 \% \mathrm{CO}_{2}$

Generation of CIK cells. Peripheral blood mononuclear cells (PBMCs) were isolated using density gradient centrifugation. The blood was separated with $650 \mathrm{x} g$ for $20 \mathrm{~min}$ at $4^{\circ} \mathrm{C}$. The CIK cells were cultured using a previously described method (19). Briefly, PBMCs were cultured in RPMI-1640 (HyClone; GE Healthcare Life Sciences, Logan, UT, USA supplemented with $10 \%$ FBS and 1,000 U/ml interferon $\gamma$ (Prospec-TanyTechnoGene, Ltd., East Brunswick, NJ, USA). Following $24 \mathrm{~h}, 50 \mathrm{ng} / \mathrm{ml}$ humanized anti-cluster of differentiation 3 monoclonal antibody (cat. no. TL-101; dilution, 1:10,000; Wuhan Institute of Biological Products, Wuhan, China) and 1,000 U/ml recombinant human interleukin-2 (rhIL-2; Prospec-TanyTechnoGene, Ltd.) were added to the cell culture. Fresh medium was added to the culture every 3 days and rhIL-2 every 6-7 days until CIK cells were harvested following 3 weeks of culture.

Reverse transcription-quantitative polymerase chain reaction $(R T-q P C R)$. RNA was isolated using TRIzol ${ }^{\circledR}$ (Invitrogen; Thermo Fisher Scientific, Inc., Waltham, MA, USA) according to the manufacturer's protocol. The RT of $1 \mu \mathrm{g}$ of RNA into cDNA was performed using SuperScript ${ }^{\mathrm{TM}}$ II Reverse Transcriptase (Invitrogen; Thermo Fisher Scientific, Inc.) and stored at $-20^{\circ} \mathrm{C}$ until use. RT-qPCR was performed with SYBR Premix Ex Taq (TransGen Biotech Co., Ltd., Beijing, China) under the following cycling conditions: one cycle at $94^{\circ} \mathrm{C}$ for $30 \mathrm{sec}, 40 \mathrm{cycles}$ at $94^{\circ} \mathrm{C}$ for $5 \mathrm{sec}$ and $60^{\circ} \mathrm{C}$ for $30 \mathrm{sec}$. The quantification of MHC class I polypeptide-related sequence A (MICA) and UL16 binding protein 2 (ULBP2), two ligands of NKG2D and GAPDH (housekeeping gene used as loading control) was performed using specific primers. The relative amount of MICA ligands' mRNA to GAPDH mRNA

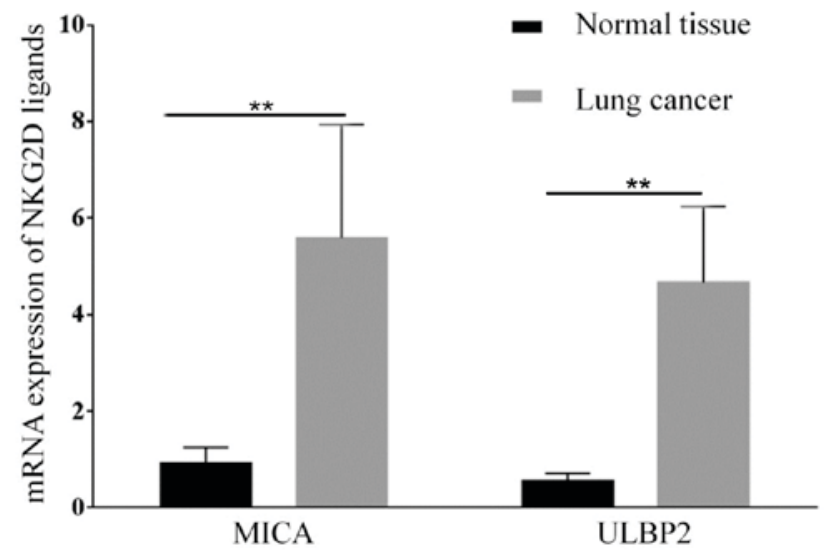

Figure 1. mRNA expression of NKG2D ligands, MICA and ULBP2, in patients with lung cancer (5 healthy tissue and 24 lung cancer tissue samples). Data are presented as the mean \pm standard deviation. ${ }^{* *} \mathrm{P}<0.01$ for lung cancer tissue vs. normal tissue (Student's t-test). NKG2D, killer cell lectin like receptor K1; MICA, MHC class I polypeptide-related sequence A; ULBP2, UL16 binding protein 2 .

(MICA/GAPDH) was used to represent the expression of the MICA genes. Analysis of relative gene expression data was analyzed using the $2^{-\Delta \Delta C q}$ methods (20). The following primers were used: MICA forward, 5'-GAGCTCCCAGCA TTCTACTAC-3'; reverse, 5'-GGTGTCGTGGCTCAAAGA TA-3'; ULBP2 forward, 5'- GAGAGGTGGTGGACATAC TTAC-3'; reverse, 5'-CAAGCCATCCTATACAGTCTCC-3'; and GAPDH forward, 5'-CTATTCGATGCCGTGTATGC-3'; reverse, 5'-GCCTGGTCCAGACTTCTTTC-3'.

Western blotting. Proteins from lung cancer and para-carcinoma tissue samples were extracted with radioimmunoprecipitation assay lysis buffer for $30 \mathrm{~min}$ and centrifuged at $13,400 \times \mathrm{g}$ for $10 \mathrm{~min}$ at $4^{\circ} \mathrm{C}$. The concentration of proteins was detected using bicinchoninic acid assay kit (Beyotime Biotechnology Co., Ltd., Shanghai, China). A total of $40 \mu \mathrm{g}$ proteins per lane were separated using 12\% SDS-PAGE gel and transferred to a polyvinylidene fluoride membrane at $100 \mathrm{~V}$ for $1 \mathrm{~h}$. The membrane was blocked using $5 \%$ non-fat dry milk for $1 \mathrm{~h}$ at room temperature to inhibit non-specific binding. Subsequently, the membrane was incubated with monoclonal rabbit anti-mouse primary antibodies directed against MICA (cat. no. ab93170; dilution, 1:500; Abcam, Cambridge, MA, USA) and $\beta$-actin (cat. no. 3700; dilution, 1:10,000; Cell Signaling Technology, Inc., Danvers, MA, USA) overnight at $4^{\circ} \mathrm{C}$. Next, membranes were incubated with horseradish peroxidase (HRP)-conjugated goat anti-rabbit IgG (cat. no. sc-2004; dilution, 1:6,000; Santa Cruz Biotechnology, Inc., Dallas, TX, USA) and peroxidase (HRP)-conjugated goat anti-mouse IgG (cat. no. sc-2005; dilution, 1:10,000; Santa Cruz Biotechnology, Inc.) secondary antibodies for $2 \mathrm{~h}$ at room temperature. The secondary antibody was detected using Pierce ${ }^{\mathrm{TM}}$ ECL Western Blotting Substrate (Thermo Fisher Scientific, Inc.).

Cytotoxicity assay. The cytotoxicity of CIK cells against lung cancer cell lines was analyzed using flow cytometry. CIK cells were used for cytotoxicity assays following 21 days of culture. Target A549 or QG56 cells were incubated with $0.1 \mu \mathrm{M}$ calcein acetoxymethyl ester (Sigma-Aldrich; Merck KGaA, 

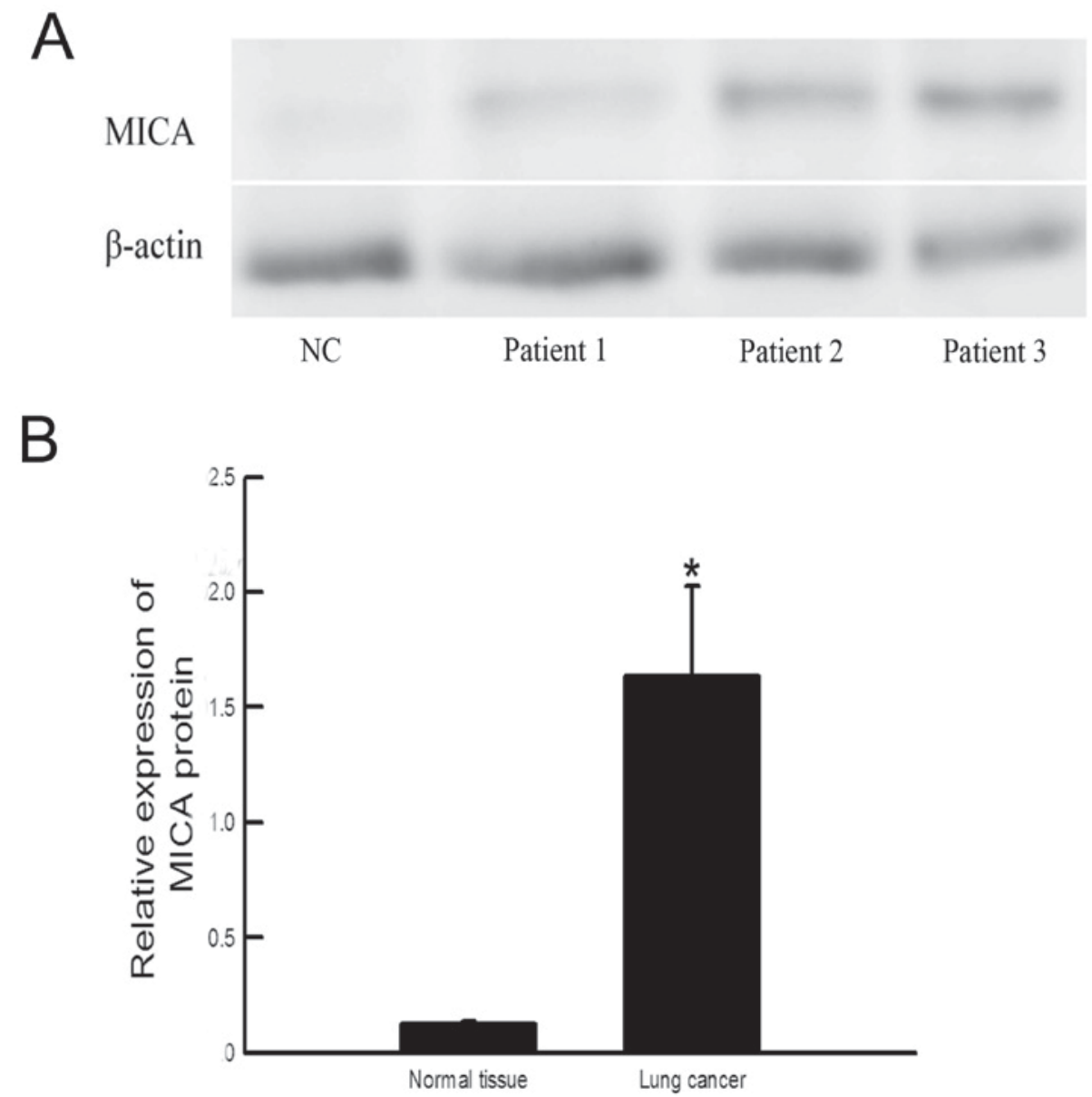

Figure 2. Expression of killer cell lectin like receptor K1 ligands in representative lung cancer tissue samples. All normal and lung cancer tissue samples are from lung cancer patients, but due to the surgical operation, not all lung cancer tissue was pare-carcinoma tissue samples. Therefore, 5 healthy and 24 lung cancer tissue samples were detected. (A) Western blot analysis of MICA in normal tis sue and lung cancer tissue. (B) Relative protein expression levels of MICA. "P<0.05 vs. normal tissue (Student's t-test). MICA, MHC class I polypeptide-related sequence; NC, normal control.

Darmstadt, Germany) at $37^{\circ} \mathrm{C}$ for $15 \mathrm{~min}$ in the dark prior to washing twice in ice-cold DMEM supplemented with $10 \%$ FBS. A total of 5,000 cells/well were re-suspended in DMEM containing $10 \%$ FBS and seeded into 96 -well plates. CIK cells were added in at distinct effect or: Target (E:T) ratios of 1, 5 and 10, in a final volume of $200 \mu 1$. The cells were incubated in the dark for $10 \mathrm{~h}$ at $37^{\circ} \mathrm{C}$ prior to washing with PBS, then cells were resuspended in $100 \mu \mathrm{l}$ of $1 \mathrm{X}$ binding buffer $(10 \mathrm{mM}$ HEPES/NaOH, $140 \mathrm{mM} \mathrm{NaCl}, 2.5 \mathrm{mM} \mathrm{CaCl}_{2}$, pH 7.4), and mixed with $10 \mu \mathrm{l}$ of 7-amino-actinomycin D (Molecular Probes; Thermo Fisher Scientific, Inc.). Cells were analyzed using flow cytometry following $15 \mathrm{~min}$ of incubation at room temperature in the dark. The cytotoxic activity of CIK against the target cells was expressed as a percentage of specific lysis as calculated using the following formula: \% specific lysis $=(\mathrm{CT}-\mathrm{TE} / \mathrm{CT}) \times 100$, where $\mathrm{CT}$ is the percentage of viable target cells in the absence of CIK cells and TE is the percentage of viable fluorescent target cells incubated with CIK cells. In blocking experiments, the CIK cells were incubated with $10 \mu \mathrm{g} / \mathrm{ml}$ anti-NKG2D antibodies (cat. no. ab203353; Abcam, Cambridge, MA, USA) at $37^{\circ} \mathrm{C}$ for $30 \mathrm{~min}$ prior to being added to the wells containing target cells.

Flow cytometry. The expression of NKG2D ligands in lung cancer cell lines was examined using flow cytometry
(BD FACSCanto $^{\mathrm{TM}}$ II; BD Biosciences, Franklin Lakes, NJ, USA) following staining with monoclonal antibodies against MICA/B (cat. no. 53-5788; dilution, 1:200; eBioscience, Inc., San Diego, CA, USA) and ULBP1, ULBP3 and ULBP2 (all R\&D Systems, Inc., Minneapolis, MN, USA) at $37^{\circ} \mathrm{C}$ for $1 \mathrm{~h}$. The data was analyzed using Flow Jo software (version 7.6; Tree Star, Inc., Ashland, OR, USA).

Statistical analysis. All statistical analyses data were presented as the mean \pm standard deviation. Student's t-tests were used to compare the differences between two groups. $\mathrm{P}<0.05$ was considered to indicate a statistically significant difference. Statistical analyses were performed using Prism software (GraphPad Software, Inc., La Jolla, CA, USA).

\section{Results}

Expression of $N K G 2 D$ ligands and ULBP2 on lung cancer tissue samples. In the present study, the expression of MICA and ULBP2, which are both ligands of NKG2D was determined in lung cancer tissue samples. The results suggest that lung cancer tissue express higher levels of MICA mRNA $(\mathrm{P}=0.003$; $\mathrm{n}=24)$ and ULBP2 $(\mathrm{P}=0.00 ; \mathrm{n}=24)$ compared with healthy tissue $(\mathrm{n}=5$; Fig. 1). The expression of MICA protein in cancer tissue was also increased compared with healthy tissue ( $\mathrm{P}=0.034$; Fig. 2). 

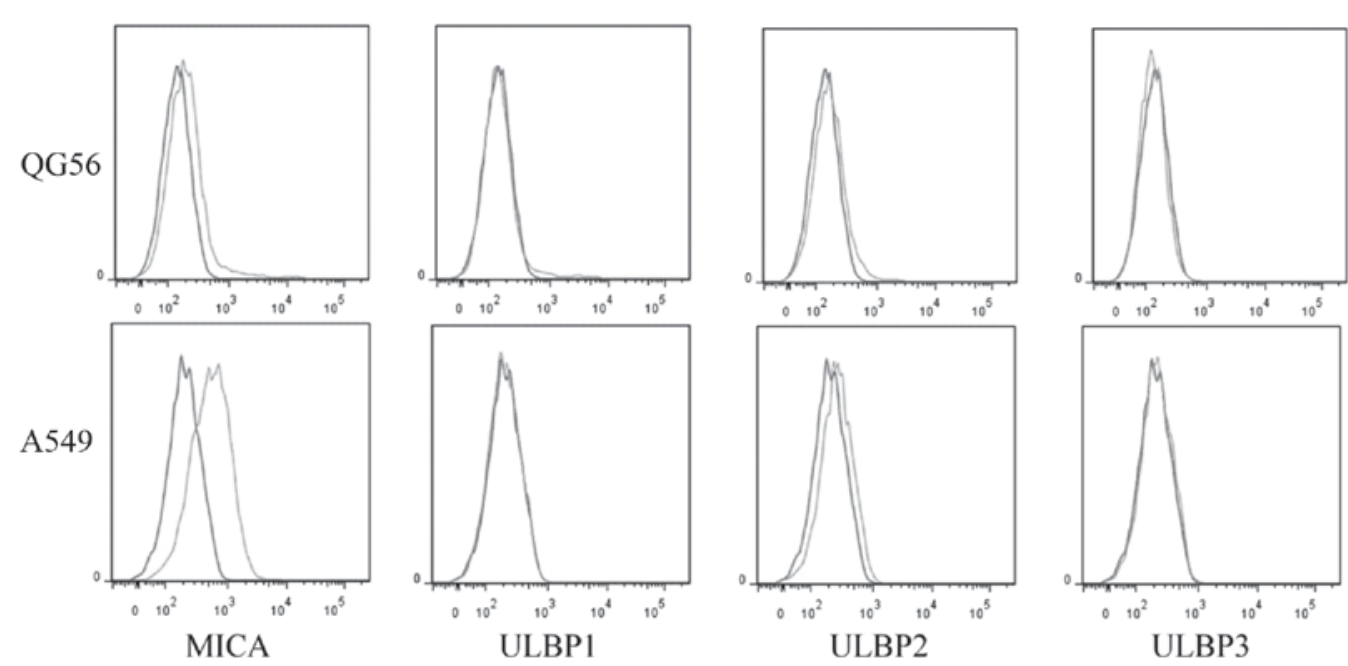

Figure 3. Expression of NKG2D ligands and human leukocyte antigen class I molecules on QG56 and A549 lung cancer cell lines. Cells were incubated with antibodies against MICA/B and ULBP1, ULBP3 and ULBP2. The data was then analyzed using Flow Jo software. NKG2D, killer cell lectin like receptor K1; MICA, MHC class I polypeptide-related sequence A; ULBP, UL16 binding protein.

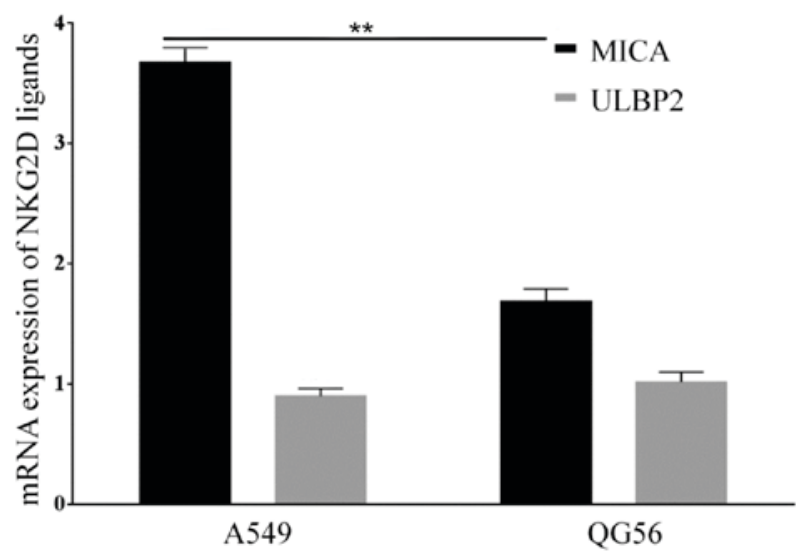

Figure 4. mRNA expression of NKG2D ligands, MICA and ULBP2 in lung cancer QG56 and A549 cell lines. Data are presented as the mean \pm standard deviation. ${ }^{* *} \mathrm{P}<0.01$ for QG56 vs. A549 cell line (Student's t-tests). NKG2D, killer cell lectin like receptor K1; MICA, MHC class I polypeptide-related sequence A; ULBP2, UL16 binding protein 2.

Expression of NKG2D ligands in lung cancer cell lines. NKG2D ligands expressed in tumor cells have previously been demonstrated to activate the anti-tumor activity of lymphocytes, with the cytotoxicity being correlated with the ratio between NKG2D ligands and (human leukocyte antigen) HLA class I molecules (11). The expression of NKG2D ligands MHC Class I molecules and ULBP son lung cancer cell lines A549 and QG56 was examined in the present study. The results suggested that both cell lines expressed MICA, but the A549 cells expressed a higher level of MICA compared with QG56 cells ( $\mathrm{P}=0.01 ; \mathrm{n}=3$; Figs. 3 and 4). However, the expression of ULBP2 between A549 and QG56 was the same (P=0.8; $n=3$; Figs. 3 and 4).

$N K G 2 D$ is involved in the CIK-mediated lysis of lung cancer cells. To investigate whether the NKG2D-NKG2D ligand interaction is involved in CIK cell-mediated recognition and cytolysis of lung cancer cells, the cytotoxic effect of CIK cells on the NKG2D ligand-expressing lung cancer A549 cell line

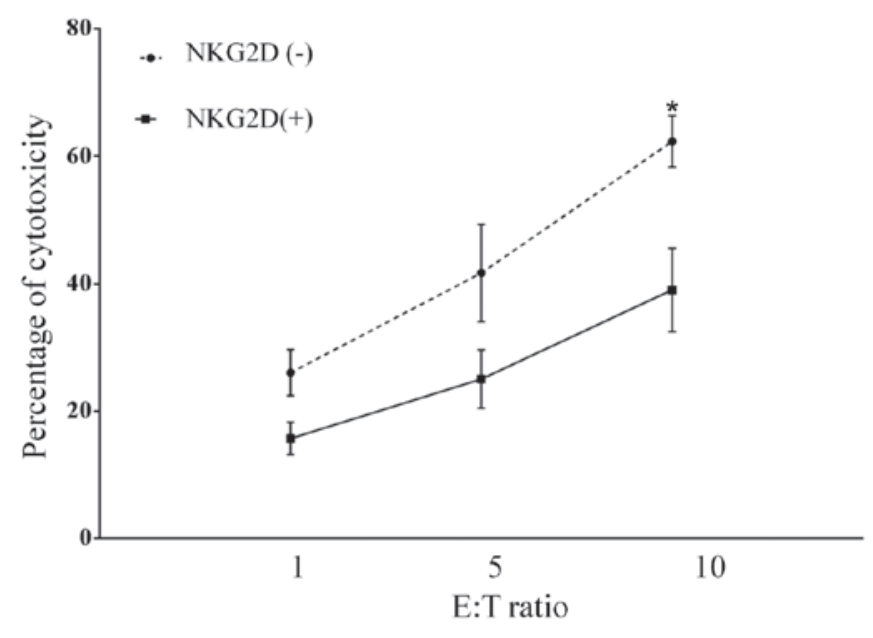

Figure 5. Involvement of NKG2D in CIK-mediated lung cancer cell lysis. The cytotoxicity effects were measured in A549 cells treated with CIK cells with and without anti-NKG2D antibody pretreatment. The percentage of cytotoxicity increased as the E:T ratio increased. The cytotoxic activity of CIK against the target cells was expressed as a percentage of specific lysis as calculated using the following formula: \% specific lysis $=(\mathrm{CT}-\mathrm{TE} / \mathrm{CT}) \times 100$, where $\mathrm{CT}$ is the percentage of viable target cells in the absence of CIK cells and TE is the percentage of viable fluorescent target cells incubated with CIK cells. Data are presented as the mean \pm standard deviation. " $\mathrm{P}<0.05$ for E:T ratio of 10 vs. E:T ratio of 1 (Student's t-test). CIK, cytokine-induced killer; NKG2D, killer cell lectin like receptor K1; E:T, effector: target ratio.

was investigated. The CIK cells caused cytolysis of A549 cells with $60 \pm 8.6 \%$ specific killing, which decreased $(30 \pm 3.2 \%)$ following pretreatment of CIK cells with anti-NKG2D antibody ( $\mathrm{P}=0.014$; Fig. 5$)$. This result suggests that the specific killing of lung cancer cells by CIK cells is partially mediated by NKG2D-NKG2D ligand interactions.

\section{Discussion}

The NKG2D-NKG2D ligand signaling pathway serves an important role in anti-tumor immunity, with NKG2D as the 
main activating receptor of NK cells that induces anti-tumor effects $(15,21)$. NKG2D ligands are expressed on the surface of tumor cells. NKG2D has been revealed to trigger natural immune cell-mediated cytotoxicity against a variety of tumor cells $(22,23)$, but NKG2D-mediated cytotoxicity requires NKG2D ligands expressed on target cells and the expression of NKG2D receptors on the surface of immune cells (11).

CIK cells are heterogeneous cells: NKG2D is expressed on the surface of all NK cells and certain T cells (24). CIK cells expressing the NKG2D receptor may be important in targeting and eliminating malignant cells. Previous data supports the notion that NKG2D ligands affect the outcomes of immune cells (11). The present study demonstrated that NKG2D ligands were expressed on the surface of lung cancer cell lines. Furthermore, that lung cancer tissue samples from patients expressed higher level of NKG2D ligands MICA and UBP2, which may serve an essential role in the recognition of lung cancer cells by immune cells. CIK cells demonstrated cytotoxicity against A549 cells in vitro, which was partially blocked by anti-NKG2D antibodies.

In conclusion, the present study has demonstrated that NKG2D ligands are expressed in lung cancer tissue samples and cell lines. The results presented suggest that the interaction between NKG2D-NKG2D ligand underlies the killing of lung cancer cells by CIK cells.

\section{Acknowledgements}

The present study was supported by Major projects foundation of Najing Medical University (grant no., 2012NJMU126).

\section{References}

1. Ferlay J, Soerjomataram I, Dikshit R, Eser S, Mathers C, Rebelo M, Parkin DM, Forman D and Bray F: Cancer incidence and mortality worldwide: Sources, methods and major patterns in GLOBOCAN 2012. Int J Cancer 136: E359-E386, 2015.

2. Schmittel A, Sebastian M, Fischer von Weikersthal L, Martus P, Gauler TC, Kaufmann C, Hortig P, Fischer JR, Link H, Binder D, et al: A German multicenter, randomized phase III trial comparing irinotecan-carboplatin with etoposide-carboplatin as first-line therapy for extensive-disease small-cell lung cancer. Ann Oncol 22: 1798-1804, 2011.

3. Jotte R, Conkling P, Reynolds C, Galsky MD, Klein L, Fitzgibbons JF, McNally R, Renschler MF and Oliver JW: Randomized phase II trial of single-agent amrubicin or topotecan as second-line treatment in patients with small-cell lung cancer sensitive to first-line platinum-based chemotherapy. J Clin Onco 29: 287-293, 2011.

4. Gerber DE and Schiller JH: Maintenance chemotherapy for advanced non-small-cell lung cancer: New life for an old idea. J Clin Oncol 31: 1009-1020, 2013.

5. Rossi A, Garassino MC, Cinquini M, Sburlati P, Di Maio M, Farina G, Gridelli C and Torri V: Maintenance or consolidation therapy in small-cell lung cancer: A systematic review and meta-analysis. Lung Cancer 70: 119-128, 2010.

6. Mesiano G, Todorovic M, Gammaitoni L, Leuci V, Giraudo Diego L, Carnevale-Schianca F, Fagioli F, Piacibello W, Aglietta $M$ and Sangiolo D: Cytokine-induced killer (CIK) cells as feasible and effective adoptive immunotherapy for the treatment of solid tumors. Expert Opin Biol Ther 12: 673-684, 2012.
7. Moretta A, Bottino C, Vitale M, Pende D, Cantoni C, Mingari MC, Biassoni R and Moretta L: Activating receptors and coreceptors involved in human natural killer cell-mediated cytolysis. Annu Rev Immunol 19: 197-223, 2001.

8. Carbone E, Neri P, Mesuraca M, Fulciniti MT, Otsuki T, Pende D, Groh V, Spies T, Pollio G, Cosman D, et al: HLA class I, NKG2D, and natural cytotoxicity receptors regulate multiple myeloma cell recognition by natural killer cells. Blood 105: 251-258, 2005.

9. Coudert JD and Held W: The role of the NKG2D receptor for tumor immunity. Semin Cancer Biol 16: 333-343, 2006.

10. Mistry AR and O'Callaghan CA: Regulation of ligands for the activating receptor NKG2D. Immunology 121: 439-447, 2007.

11. Fuertes MB, Girart MV, Molinero LL, Domaica CI, Rossi LE, Barrio MM, Mordoh J, Rabinovich GA and Zwirner NW: Intracellular retention of the NKG2D ligand MHC class I chain-related gene $\mathrm{A}$ in human melanomas confers immune privilege and prevents NK cell-mediated cytotoxicity. J Immunol 180: 4606-4614, 2008.

12. Kloss M, Decker P, Baltz KM, Baessler T, Jung G, Rammensee HG, Steinle A, Krusch M and Salih HR: Interaction of monocytes with NK cells upon Toll-like receptor-induced expression of the NKG2D ligand MICA. J Immunol 181: 6711-6719, 2008.

13. Lu X, Ohata K, Kondo Y, Espinoza JL, Qi Z and Nakao S: Hydroxyurea upregulates NKG2D ligand expression in myeloid leukemia cells synergistically with valproic acid and potentially enhances susceptibility of leukemic cells to natural killer cell-mediated cytolysis. Cancer Sci 101: 609-615, 2010.

14. Kato N, Tanaka J, Sugita J, Toubai T, Miura Y, Ibata M, Syono Y, Ota S, Kondo T, Asaka M and Imamura M: Regulation of the expression of MHC class I-related chain A, B (MICA, MICB) via chromatin remodeling and its impact on the susceptibility of leukemic cells to the cytotoxicity of NKG2D-expressing cells. Leukemia 21: 2103-2108, 2007.

15. Bae DS, Hwang YK and Lee JK: Importance of NKG2D-NKG2D ligands interaction for cytolytic activity of natural killer cell. Cell Immunol 276: 122-127, 2012.

16. Pende D, Rivera P, Marcenaro S, Chang CC, Biassoni R Conte R, Kubin M, Cosman D, Ferrone S, Moretta L and Moretta A: Major histocompatibility complex class I-related chain A and UL16-binding protein expression on tumor cell lines of different histotypes: Analysis of tumor susceptibility to NKG2D-dependent natural killer cell cytotoxicity. Cancer Res 62: 6178-6186, 2002.

17. Friese MA, Platten M, Lutz SZ, Naumann U, Aulwurm S, Bischof F, Bühring HJ, Dichgans J, Rammensee HG, Steinle A and Weller M: MICA/NKG2D-mediated immunogene therapy of experimental gliomas. Cancer Res 63: 8996-9006, 2003.

18. Salih HR, Antropius H, Gieseke F, Lutz SZ, Kanz L, Rammensee HG and Steinle A: Functional expression and release of ligands for the activating immunoreceptor NKG2D in leukemia. Blood 102: 1389-1396, 2003.

19. Linn YC, Lau SK, Liu BH, Ng LH, Yong HX and Hui KM: Characterization of the recognition and functional heterogeneity exhibited by cytokine-induced killer cell subsets against acute myeloid leukaemia target cell. Immunology 126: 423-435, 2009.

20. Livak KJ and Schmittgen TD: Analysis of relative gene expression data using real-time quantitative PCR and the 2(-Delta Delta C(T)) Method. Methods 25: 402-408, 2001.

21. Morisaki T, Onishi $\mathrm{H}$ and Katano M: Cancer immunotherapy using NKG2D and DNAM-1 systems. Anticancer Res 32: 2241-2247, 2012.

22. Zafirova B, Wensveen FM, Gulin M and Polić B: Regulation of immune cell function and differentiation by the NKG2D receptor. Cell Mol Life Sci 68: 3519-3529, 2011.

23. Champsaur M and Lanier LL: Effect of NKG2D ligand expression on host immune responses. Immunol Rev 235: 267-285, 2010.

24. Spear P, Wu MR, Sentman ML and Sentman CL: NKG2D ligands as therapeutic targets. Cancer Immun 13: 8, 2013. 